\title{
Radio Spectrum Valuation by Using Censored Regression Method
}

\author{
Settapong Malisuwan, Noppadol Tiamnara, Nattakit Suriyakrai \\ National Broadcasting and Telecommunications Commission (NBTC), Bangkok, Thailand \\ Email: settapong.m@nbtc.go.th,noppadol.t@nbtc.go.th,nattakit.s@nbtc.go.th
}

Received 8 October 2015; accepted 8 November 2015; published 11 November 2015

Copyright $@ 2015$ by authors and Scientific Research Publishing Inc.

This work is licensed under the Creative Commons Attribution International License (CC BY). http://creativecommons.org/licenses/by/4.0/

(c) $\underset{\mathrm{EY}}{\mathrm{E}}$ Open Access

\begin{abstract}
Currently, the mobile markets in every country all around the world have been developing rapidly. The adoption of 3G and 4G services will greatly benefit the economy. Consumers will receive fast and quality telecommunication system and their quality of life will improve. Such development will be beneficial to the production of goods and service in countries raising overall competitiveness both domestically and internationally. Radio spectrum is scarce and invaluable telecommunication resource. Spectrum auction should determine spectrum value as consistent to its actual value. In order to ensure that spectrum is efficiently assigned, spectrum is priced to reflect the value it can add to help promote economic and technical efficiency with users who have bid for it. Putting a price on spectrum not only ensures spectrum management efficiency, but also can add revenue for the government which will in turn cover the cost of spectrum. This paper presents a radio spectrum valuation method by using censored regression method. The contributions in this paper could assist telecom policy makers to gain more understanding in development of radio spectrum valuation.
\end{abstract}

\section{Keywords}

Radio Spectrum, Valuation, Censored Regression, Estimation

\section{Introduction}

Studies on telecommunication service spectrum valuation were not widespread in the past but becoming more readily available after the year 2000. From literature review on the spectrum valuation studies from international experts, we can categorize the studies into two groups [1]. The first values spectrum by opportunity cost approach, which compares the cost and revenue of bidders with and without spectrum. The second group of studies applies econometrics to data collected from past auctions in different countries as a guideline in spectrum valua- 
tion for studied country. It starts by using statistical technique to estimate regression model in order to find the relationship between actual auction prices in different countries and its determinants. Then, it forecasts the spectrum price for studied country by replacing the values of determinants in the equation. In this paper, we focus on the second group which uses economic method in finding the relationship between spectrum price from real auctions in different countries (auction price) and its determinants. Spectrum valuation by econometrics is easier than the opportunity cost approach as an econometric model needs macro-level data which are usually public. In contrast, the calculation of opportunity costs needs micro-level data including engineering, technology and business data which are usually confidential.

This paper presents a radio spectrum valuation method by using censored regression method which is a type of econometrics method. To construct censored regression model, the study in [2] categorized determinants of spectrum value (price/MHz/pop) into five groups as shown in Table 1: variables that reflected economic condition and mobile market characteristics, license characteristics, financial obligation, service obligations and auction procedure.

Most variables on economic condition and mobile market characteristics have significant impacts on spectrum value. The study found that the auction price would be higher if the population coverage of the spectrum license increased. This result was consistent with [3] who conducted a theoretical study from both engineering and economic perspectives, and empirical study to test the result using the data from spectrum auctions in Europe. It found that in theory, important determinants were spectrum demand represented by traffic and spectrum supply represented by bandwidth. In the empirical research, it found that the key determinants of spectrum value were the number of populations (users). However, the spectrum value has a nonlinear relationship with the number of populations. In addition, the income per capita also has a positive impact on spectrum price.

In the license characteristics group of variables, the license period and the reserve price are two factors that have significant impacts on spectrum value. These two variables have a positive impact on spectrum value. The findings were consistent with [1] [4]-[6] which found that the sufficiently high reserve price prevented bidder collusion, which kept the auction price low.

Table 1. Determinants of spectrum value in study of [2].

\begin{tabular}{|c|c|c|}
\hline Key variables group & Variable & Description \\
\hline \multirow{5}{*}{$\begin{array}{l}\text { Economic condition and mobile } \\
\text { market characteristics }\end{array}$} & Income & Real income per capita (US Dollar in PPP) \\
\hline & Market & Population in coverage area of spectrum license (million) \\
\hline & Mcomp & Reciprocal of one plus the number of operators \\
\hline & Shift (dummy) & Equal to one if auction took place during 2001 to 2007, zero otherwise \\
\hline & Duration & License period (year) \\
\hline \multirow[t]{2}{*}{ License characteristics } & Entrant (dummy) & $\begin{array}{l}\text { Equal to one if auctions rule stipulates that at least one } \\
\text { license is set-aside for operator who has never } \\
\text { received spectrum license, zero otherwise }\end{array}$ \\
\hline & Reserve & Reserve price (US dollar) \\
\hline $\begin{array}{l}\text { Financial commitment after } \\
\text { winning the auction }\end{array}$ & Percent & Means of annual fee (percent of income from using 3G spectrum) \\
\hline \multirow{2}{*}{$\begin{array}{l}\text { Service obligation after winning } \\
\text { the auction }\end{array}$} & Deploy & Percent of population in accordance with coverage obligation \\
\hline & Share (dummy) & Equal to one if infrastructure sharing is mandated \\
\hline \multirow{6}{*}{ Auction procedure } & Accomp & Ratio of the number of bidders to the number of license \\
\hline & Activity (dummy) & Equal to one if auction has activity rule, zero otherwise \\
\hline & Info (dummy) & $\begin{array}{l}\text { Equal to one if auction information are } \\
\text { disclosed to the public, } 0 \text { otherwise }\end{array}$ \\
\hline & Number (dummy) & Equal to one if the number of license is predetermined, zero otherwise \\
\hline & Package (dummy) & Equal to one if package bidding is permitted, zero otherwise \\
\hline & Sealed (dummy) & Equal to one if auction is a sealed-bid format, zero otherwise \\
\hline
\end{tabular}


According to the studies of spectrum valuation by censored regression in the past, we can categorize variables that have significant effects on spectrum value and have the relationship as predicted by the theory into three groups: 1) factors concerning spectrum demand, 2) factor concerning spectrum auction characteristics and 3) factors concerning pre- and post-auction obligations. Factors concerning spectrum demand are variables that reflect the size of the economy and the needs for spectrum. This group includes country's income, the number of populations, and the time when telecommunication market is booming. The group of factors concerning spectrum auction characteristics will express the level of competition in spectrum auction, including the number of bidders per license offered. The last group of variables includes factors concerning pre- and post-auction obligations. They are important factors to be considered by bidders before entering the auction. These include reservation of license for next auction, amount of deposit to be made before auction, annual fee and coverage obligation.

\section{Spectrum Valuation with Economic Approach: Censored Regression Method}

Spectrum valuation with econometric approach considers demand- and supply-side determinants of spectrum value. Supply-side determinants are, for example, license size, total spectrum to be auctioned, license length and set-aside. Demand-side determinants are GDP, GDP per capita, the number of subscribers, education level, ratio of revenue of telecommunication sector to GDP and long-run technological change. In short, spectrum valuation with econometric approach is to find determinants of spectrum value while considering spectrum packaging, licensing procedure, payment schedule, domestic economy and market condition.

There are some remarks about the estimations. First, to specify a model, the independent variables must be chosen in accordance with the theory or sensible assumptions. International Telecommunication Union (ITU) categorizes determinants of spectrum value [7] [8].

Nevertheless, there is no rule about the choice of independent variables. It depends on data availability, assumptions, historical data and economic theory. Each of these criteria may be applied individually or together when choosing independent variables. It can say that an econometric model specification is both arts and science.

Second, data for the study are collected from government agencies, domestic and international firms and other sources such as auction news. In practice, some variables cannot be included because of several reasons (e.g. no data available). Therefore, some dependent or independent variables have to be replaced with other substitutable variables that have available data.

Third, the model estimation is to estimate coefficients that explain the relationship between the spectrum value and the selected independent variables. There are two estimation techniques, ordinary least squares and maximum likelihood estimation.

In this paper, we consider maximum likelihood estimation which is the censor regression model. Since spectrum value may be unobservable n some case, so-called censored sample, a censored regression model is used to solve such problem. The censored regression model is estimated with maximum likelihood technique.

The censored regression model can be written as

$$
Y_{i}^{*}=X^{\prime} \beta+\varepsilon_{i}
$$

where $Y^{*}$ is the index of spectrum value indicating that spectrum value is observable (uncensored sample) or observable (censored sample) data. $x$ is a vector of independent variables. $\beta$ is a vector of coefficients to be estimated. $\varepsilon$ is errors between the actual and predicted spectrum value. The index is

$$
Y_{i}=f(x)= \begin{cases}Y_{i}^{*}, & Y_{i}^{*}<\tau \\ 0, & Y_{i}^{*} \geq \tau\end{cases}
$$

where $\tau$ is the critical value designating whether spectrum value is an uncensored or censored sample, $X$ is a vector of independent variables, $\beta$ is a vector of coefficients and $\varepsilon$ are errors between actual and predicted spectrum values.

The censored regression model is estimated with maximum likelihood. Conceptually, the distribution of spectrum value $Y$ is a normal distribution that takes into account unobservable value as shown in the following equation.

$$
f(Y)=\left[f\left(Y^{*}\right)\right]^{d_{i}}[F(\tau)]^{1-d_{i}}
$$


where the maximum likelihood estimation considers the probability that each sample is observed to construct the likelihood function and estimate the parameters. The likelihood function can be written as follows.

$$
L=\prod_{i}^{N}\left[\frac{1}{\sigma} \varnothing\left(\frac{Y_{i}-X_{i} \beta}{\sigma}\right)\right]^{d_{i}}\left[1-\varnothing_{c}\left(\frac{Y_{i}-X_{i} \beta}{\sigma}\right)\right]^{1-d_{i}}
$$

Taking log to get the log-likelihood function

$$
\max _{\beta, \sigma^{2}} \ln (L)=\sum_{i=1}^{N}\left[d_{i}\left(-\ln \sigma+\ln \varnothing\left(\frac{Y_{i}-X_{i} \beta}{\sigma}\right)\right)+\left(1-d_{i}\right) \ln \left(1-\varnothing_{c}\left(\frac{Y_{i}-X_{i} \beta}{\sigma}\right)\right)\right]
$$

After estimating the censored regression model, if the sample is sufficiently large so that its distribution is approximately normal, the estimators will be unbiased. Reliable estimates lead to reliable spectrum valuation. Therefore, the censored regression model is one of the most popular models for spectrum valuation if a sufficiently large dataset is available.

In sum, econometrics is an important tool for analyzing economic data as econometrics combines mathematics, statistics and economic theory to construct a mathematical model to explain the relationship between the dependent variable and its determinants given historical data. This model is not only able to portray influences of independent variables on the dependent variable, but it can forecast the value of the dependant variable as well.

\section{Analysis of Spectrum Valuation Data Characteristics}

Spectrum auction can be viewed as investment and a firm wants to invest in a profitable project. If awarded a spectrum license, a firm can make use of spectrum not only to improve its telecommunication service, but to reduce cost of build out as well. A firm would participate in a spectrum auction whenever the net revenue from spectrum license exceeds the auction price.

However, in each auction, government or auctioneer usually sets a reserve price for the auction. The reserve price is usually greater than zero. The winning bidder is the one submitted the highest bid given that it must be greater than the reserve price.

If the net revenue from spectrum license is less than the reserve price, firms will not participate in the auction and the winning bid is equal to zero. Nevertheless, spectrum value is not equal to zero but it is simply lower than the reserve price. Therefore, some auction prices are not observable and the data are thereby a censored sample. The least squares are not appropriate for spectrum valuation because the estimates will be higher than the actual values. An appropriate model for censored sample is Tobit.

Developed by James Tobin, Tobit model is suitable for a sample whose data for dependent variable is censored or censored sample.

The study in [9] employed Tobit model to estimate the relationship between spectrum value in unit of price/ $\mathrm{MHz} /$ pop and its determinants. They argued that winning bids are typically censored since an observable winning bid must be the highest bid that is greater than the reserve price. In some circumstances, there are no bids as the return from spectrum license is less than the reserve price. As a result, there is no winning bidder and his spectrum value is unobservable. The winning bid is then equal to zero.

Due to such winning bid data, the spectrum value cannot be estimated with the least squares. Tobit model which takes missing data of the dependent variable into consideration is more suitable for spectrum valuation.

This section discusses how to estimate Tobit model with maximum likelihood under assumption that variables are distributed normally. The probability distribution function and cumulative distribution function of normal variables are shown first and then the estimation of Tobit model is discussed.

Let $y$ be a normal random variable with the mean of $\mu$ and the variance of $\sigma^{2}$. Thus, the probability distribution function of $y$ is

$$
f(y)=\frac{1}{\sqrt{2 \pi \sigma^{2}}} \mathrm{e}^{-\frac{(y-\mu)^{2}}{2 \sigma^{2}}}
$$


The standard normal distribution of $y$ with the mean of 0 , the variance of 1 and $z=\frac{y-\mu}{\sigma}$ is

$$
f(y)=\varnothing\left(\frac{y-\mu}{\sigma}\right)=\varnothing(z)=\frac{1}{\sigma \sqrt{2 \pi}} \mathrm{e}^{-\frac{1}{2} \cdot \frac{(y-\mu) \cdot(y-\mu)}{\sigma} \cdot \frac{1}{\sigma \sqrt{2 \pi}}} \mathrm{e}^{\left(-\frac{z^{2}}{2}\right)}
$$

When $\sigma=1$,

$$
f(z)=\varnothing(z)=\frac{1}{\sqrt{2 \pi}} \mathrm{e}^{\left(-\frac{z^{2}}{2}\right)}
$$

Let $\varnothing(z)$ is the standard normal distribution function

$\varnothing_{c}(z)$ is the cumulative standard normal distribution function. Thus,

$$
f(y)=\frac{1}{\sqrt{2 \pi \sigma^{2}}} \mathrm{e}^{-\frac{(y-\mu)^{2}}{2 \sigma^{2}}}=\frac{1}{\sigma \sqrt{2 \pi}} \mathrm{e}^{\left(-\frac{z^{2}}{2}\right)}=\frac{1}{\sigma} \varnothing\left(\frac{y-\mu}{\sigma}\right)=\frac{1}{\sigma} \varnothing(z)
$$

where the cumulative distribution function is

$$
\begin{aligned}
& p(Y \leq y)=\varnothing_{c}\left(\frac{y-\mu}{\sigma}\right) \\
& p(Y>y)=1-\varnothing_{c}\left(\frac{y-\mu}{\sigma}\right)
\end{aligned}
$$

As discussed earlier, the winning bid price per total MHz/pop data are left-censored sample since a firm decides not to bid if the net revenue from spectrum license is below the reserve price. As a result, the winning bid is equal to zero. Nevertheless, the spectrum value is not equal to zero. It is rather equal to the return which is below the reserve price. This value is unobservable as shown in Figure 1.

According to Figure 1, the censored sample distribution can be written as follows.

$$
P M H z p_{i j}= \begin{cases}P M H z p_{i j}^{*} & \text { if } P M H z p_{i j}^{*}>\text { Reserve } \text { price }_{i j} \\ 0 & \text { if } P M H z p_{i j}^{*} \leq \text { Reserve price }_{i j}\end{cases}
$$

Let $y_{i j}^{*}$ be the highest bid which is initially confidential and thus unobserved. This value is revealed only if it is greater than the reserve price and it is the highest as in shown in the first case of $P M H z p_{i j}$. The second case involves no bid submitted since the spectrum value is below the reserve price and it is thereby not revealed and equal to zero. Hence, the distribution of $P M H z p$ is normal with unobserved data as shown in the following equation.

$$
f(\text { PMHzp })=\left[f\left(\text { PMHzp }^{*}\right)\right]^{d_{i}}[F(\text { Reserve price })]^{1-d_{i}}
$$

where $d_{i}=1$ if $P M H z p_{i j}^{*}>$ Reserve price ${ }_{i j}$;

$d_{i}=0$ if $P M H z p_{i j}^{*} \leq$ Reserve price $_{i j}$.

So, the cumulative distribution function of the censored data is

$p($ Censored $)=p\left(P M H z p_{i j}^{*} \leq\right.$ Reserve price $\left._{i j}\right)=\varnothing_{c}\left(\frac{\text { Reserve price }_{i j}-\mu}{\sigma}\right)=1-\varnothing_{c}\left(\frac{\mu-\text { Reserve } \text { price }_{i j}}{\sigma}\right)$

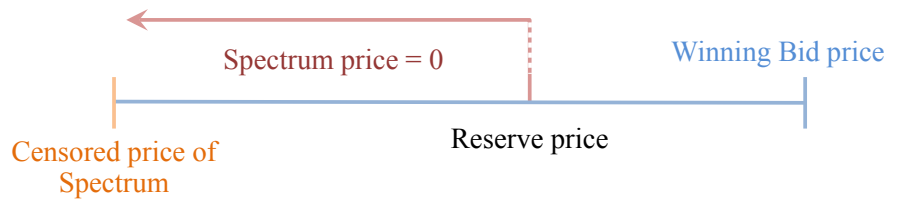

Figure 1. Left-censored spectrum value. 
The cumulative distribution function of censored data is shown in Figure 2.

The cumulative distribution function is the white area in Figure 2. If the distribution is assumed uncensored despite the imperfect data, the statistical properties violate the least squares assumptions and the estimation result is biased and inconsistent.

Tobit model uses the probability above for estimation and thus requires assumption about the distribution of the random variable. It is usually assumed to be a normal distribution.

After showing the probability distribution of uncensored and censored data, estimation of Tobit model with maximum likelihood which pools both uncensored and censored data as in probability theory is discussed.

Even if Tobit model specifies a linear relationship between dependent and independent variables as in the general linear regression, some censored sample cannot be observed and thus the dependent variable is equal to zero. The dependent variable, spectrum value $(\mathrm{PMHz})$, is specified as $\mathrm{PMHz}^{*}$ under a condition that $\mathrm{PMHz}^{*}$ can be observed only when $P M H z^{*}$ is greater than the reserve price and it is the highest among all bidders. However, $P \mathrm{MHz}^{*}$ is unobservable if a firm value the spectrum less than the reserve price. As a result, there is no winning bidder and the spectrum value is equal to zero as discussed earlier. Therefore, the Tobit regression model can be written as follows.

$$
P M H z p_{i j}^{*}=X_{i j} \beta+\varepsilon_{i j}
$$

where $P M H z p_{i j}^{*}$ is spectrum value per $\mathrm{MHz} /$ pop under sample observability conditions;

$x^{\prime}$ is the vector of determinants of spectrum value per MHz/pop;

$\varepsilon$ captures factors other than the determinants of spectrum value. Normally, it is assumed to be negligible in explaining spectrum value;

$i$ and $j$ are subscripts representing country and license index, respectively. The value of $P M H z p^{*}$ is specified as follows.

$$
P M H z p_{i j}= \begin{cases}P M H z p_{i j}^{*} & \text { if } P M H z p_{i j}^{*}>\text { Reserve } \text { price }_{i j} \\ 0 & \text { if } P M H z p_{i j}^{*} \leq{\text { Reserve } \text { price }_{i j}}^{*}\end{cases}
$$

The estimation of Tobit model which considers censored sample is shown below. The distribution of $\mathrm{PMHz}$ follows a normal distribution with censored data as shown below.

$$
f(\text { PMHzp })=\left[f\left(\text { PMHzp }{ }^{*}\right)\right]^{d_{i}}[F(\text { Reserve price })]^{1-d_{i}}
$$

where $d_{i}=1$ if $P M H z p_{i j}^{*}>$ Reserve price ${ }_{i j}$

$d_{i}=0$ if $P M H z p_{i j}^{*} \leq$ Reserve price $_{i j}$.

Maximum likelihood estimation uses probabilities of each realization to construct a likelihood function to estimate parameters. The likelihood function is written as follows.

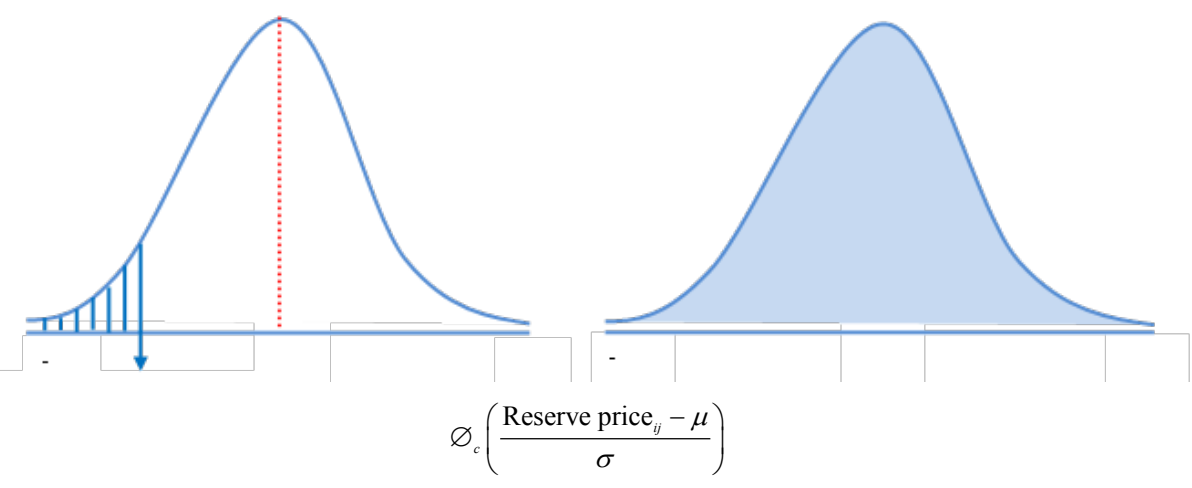

Figure 2. Comparison between cumulative distribution functions of censored data (left) and uncensored data (right), The cumulative distribution function of uncensored data is $p($ Uncensored $)=1-\varnothing_{c}\left(\frac{\text { Reserve } \text { price }_{i j}-\mu}{\sigma}\right)$. Note: reserve price is smaller than the winning bid price. 


$$
L=\prod_{i}^{N}\left[\frac{1}{\sigma} \varnothing\left(\frac{P M H z p_{i j}-X_{i j} \beta}{\sigma}\right)\right]^{d_{i}}\left[1-\varnothing_{c}\left(\frac{X_{i j} \beta-\text { Reserve price }_{i j}}{\sigma}\right)\right]^{1-d_{i}}
$$

Taking log yields a log-likelihood function

$\operatorname{Max}_{\beta, \sigma^{2}} \ln (L)=\sum_{i=1}^{N}\left[d_{i}\left(-\ln \sigma+\ln \varnothing\left(\frac{P M H z p_{i j}-X_{i j} \beta}{\sigma}\right)\right)+\left(1-d_{i}\right)\left(\ln 1-\varnothing_{c}\left(\frac{X_{i j} \beta-\text { Reserve price }_{i j}}{\sigma}\right)\right)\right]$

The log-likelihood function consists of two terms. The first term is the estimation of uncensored data and the second term is the additional condition for estimation, says the probability that the sample is censored. Therefore, the estimation of Tobit model not only considers observable data, but it estimates parameters that are consistent to censored data as well.

The predicted spectrum value from the Tobit model is non-linear as shown in the equation below.

$$
E\left(P M H z p_{i j} \mid X_{i j}\right)=\varnothing_{c}\left(\frac{X_{i j} \beta}{\sigma}\right)\left(X_{i j} \beta+\sigma \varphi_{i j}\right)
$$

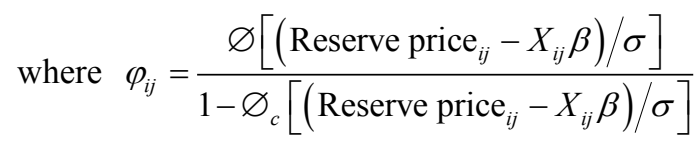

The marginal effect considers a change in determinant of spectrum value. In other words, it considers the magnitude and direction of change in spectrum value when an determinant of spectrum value increases by one unit. The marginal effect can be calculated as follows.

$$
\frac{\partial E\left(P M H z p_{i j} \mid X_{i j}\right)}{\partial x_{i j}}=\beta
$$

Pros and Cons of Tobit Model

Pros. Since Tobit model requires maximum likelihood estimation, given that the sample size is sufficiently large, Tobit model is desirable for estimating with variables or data that are inaccessible, especially spectrum auction in which only winning bid prices are observed.

Cons. Tobit model requires maximum likelihood estimation assuming that variables are distributed normally. In practice, according to the Central Limit Theorem, a sufficiently large sample is needed to overcome the problem of unknown distribution. Hence, if the sample size is small, the estimation will be inaccurate and inconsistent. This is different from OLS which does not assume any particular distribution.

\section{Estimation Framework}

A good framework for estimation and prediction should have the following properties.

Variables in the model must be sensible and have precise conceptual foundation. In this study, we apply idea from ITU and economic concepts as a basis for variable selection. However, ITU determinants of spectrum value rely on theoretical concepts. For some determinants, quantitative data are not available and their proxies are used instead and for others, there is no data available. Nevertheless, from the precise theoretical concept, we can conjecture the effects of various variables on spectrum value, whether they are positive or negative. Therefore, a desirable model should yield the estimation result consistent with the expectation.

The predicted value from the model must be sensible and practical. Specifically, the predicted value must not be unusually low or high. Also, it must not be insensible value such as negative spectrum value.

Statistical properties should be reliable. For censored regression, we consider two statistics: 1) significant level of various variables along with their signs, whether they are the same as expected, 2) pseudo R-squared which is a statistic used for comparing explanatory powers (spectrum value) of different models. The pseudo Rsquared can be used in similar way as R-squared in least squares estimation. That is, a model with higher pseudo $\mathrm{R}$-squared can explain spectrum value statistically better. However, in the censored regression, R-squared cannot be calculated. Thus, pseudo R-squared is used instead. The value of pseudo R-squared is not restricted to be between 0 and 1 as R-squared from OLS but the better model has a higher pseudo R-squared as in the general 
linear regression.

\section{Conclusion}

This paper aims to present the radio spectrum valuation by using censored regression model which is an econometric model. Basically, Radio spectrum valuation employs econometric models and methodologies which integrate mathematics, statistics, and economic theory to model the relationship between spectrum value and its determinants. Econometrics has several advantages. First, it studies actual historical data instead of forecasted data which are usually associated with uncertainty. Second, econometrics is desirable for the available dataset. Furthermore, econometrics is more flexible and tractable. This allows us to appropriately define the relationship between spectrum value and its determinants as well as better predict the spectrum value. Lastly, there are diverse econometric methods and techniques.

\section{References}

[1] Cramton, P. (2002) Spectrum Auctions.

[2] Madden, G., Sağlam, I. and Morey, A. (2010) Auction Design and the Success of National 3G Spectrum Auctions. TÜSİAD-Koç University Economic Research Forum Working Paper Series.

[3] Van Bruwaene, K. (2003) Estimating the Value of Spectrum. EBU Technical Review.

[4] Klemperer, P. (2002) What Really Matters in Auction Design. The Journal of Economic Perspectives, 16, 169-189. http://dx.doi.org/10.1257/0895330027166

[5] Cave, M.E., Majumdar, S.K. and Vogelsang, I. (2003) Handbook of Telecommunications Economics. Info, 5, 46-46.

[6] Cramton, P. (2006) Simultaneous Ascending Auctions. Wiley Encyclopedia of Operations Research and Management Science.

[7] Alden, J. (2012) Exploring the Value and Economic Valuation of Spectrum. International Telecommunication Union, Geneva.

[8] Dippon, C.M. (2009) Regulatory Policy Goals and Spectrum Auction Design: Lessons from the Canadian AWS Auction. NERA Economic Consulting, Statement.

[9] Bohlin, E., Madden, G. and Morey, A. (2010) An Econometric Analysis of 3G Auction Spectrum Valuations. 